\title{
Knowledge, Attitudes, Beliefs and Practices on HIV/AIDS among Boatmen on the Maroni River: A Neglected Bridging Group?
}

\author{
Emilie Gaubert-Marechal ${ }^{\star \star}$, Anne Jolivet ${ }^{2,3,4}$, Astrid Van-Melle ${ }^{5}$, Marie-Claire Parriault ${ }^{5}$, Célia Basurko ${ }^{5}$, Antoine Adenis ${ }^{5}$, Mathieu \\ Hanf $^{5}$,Vincent Vantilcke ${ }^{2}$, Sandrine Halfen ${ }^{6}$, Pierre Couppie ${ }^{7,8}$ and Mathieu Nacher ${ }^{1,5,7}$ \\ ${ }^{1}$ COREVIH Guyane, General Hospital Andrée Rosemon, Cayenne, French Guiana, France \\ ${ }^{2}$ Department of Medicine, General Hospital Franck Joly, Saint-Laurent du Maroni, French Guiana, France \\ IINSERM, U707, Research Team on the Social Determinants of Health and Health Care, Paris, France \\ ${ }^{4}$ Université Pierre et Marie Curie, Paris 06, UMR-S 707, Paris, France \\ ${ }^{5}$ Centre of Clinical Investigation Epidémiologie Clinique Antilles-Guyane, CIC-EC INSERM CIE 802, General Hospital Andrée Rosemon, Cayenne, French Guiana, France \\ ${ }^{6}$ Regional Observatory of the Health of Ile-de-France, Paris, France \\ ${ }^{7}$ UFR of medicine, Université Antilles-Guyane, France \\ ${ }^{8}$ Department of Dermatology, General Hospital Andrée Rosemon, Cayenne, French Guiana, France
}

\begin{abstract}
In French Guiana, the prevalence of HIV among pregnant women along the Maroni river, has risen from 0 to over $1 \%$ in the 1990's. Despite this sharp increase, no behavioural surveillance study has ever taken place in this border region. The present study showed that an important proportion of boatmen have risky sexual behaviour. These results underline the importance of targeting prevention interventions on this population that is not currently receiving specific HIV prevention messages.
\end{abstract}

Keywords: HIV; Behaviour; French Guiana; Boatmen

\section{Introduction}

French Guiana, located in the north-eastern part of the South American continent with a population of 224469 inhabitants in 2009. It is the French Guiana is located territory that is most affected by the HIV epidemic. With a prevalence of HIV among pregnant women $>1 \%$, the epidemic there is generalised according to UNAIDS. However, its drivers have not been well described. Recently, the roles of sex work and crack have been suggested [1]. However, there are great differences between Cayenne, the main city, and western French Guiana. The Maroni river traces the border between French Guiana and Suriname over a distance of $520 \mathrm{~km}$ cutting through the Amazon forest. It represents one of the main access routes towards villages in the interior for persons and goods transportation. The population living in the villages on the French side of the Maroni River, excluding the city of St-Laurent du Maroni, can be estimated at 22644 inhabitants in 2009. In these villages, the HIV prevalence among pregnant women has increased with a 10 year lag compared to the rest of French Guiana, rising from $0 \%$ in the early 1990 s to $1.1 \%$ in 2003 , and remaining above $1 \%$ since then [1]. Although, qualitative studies have suggested that persons living on the Maroni, mostly Maroon populations were at risk for HIV [2], no quantitative behavioural study has ever been conducted there. Migrations and population mobility are considered HIV risk factors [3,4]. We hypothesized that the Maroni boatmen, being highly mobile, could therefore have a high risk of HIV infection and subsequently transmitting it [3]. Thus, the principal objective of this study was to describe the knowledge, attitudes, beliefs and practice regarding HIV/AIDS among boatmen on the Maroni and to compare the results with those of the general population of French Guiana coast [5].

\section{Methods}

An anonymous cross sectional observational Knowledge Attitudes Beliefs and Practices (KABP) study was conducted on Maroni boatmen. The target population was boatmen aged 16-69 years working on the Maroni river between June and October 2010. Street outreach recruitement of boatmen used at all the landing sites in Saint Laurent $\mathrm{du}$ Maroni were investigated. The landing sites were la Charbonière,
Village chinois, Balaté, Paddock and la Glacière. The data collection was anonymous and the goals of the research were explained to all boatmen interviewed who gave informed consent to answer a structured questionnaire exploring sociodemographic characteristics and KABP regarding HIV. More specifically the questionnaire explored: knowledge of transmission routes, knowledge of protection strategies, first intercourse, sexual activity, recourse to paid sex, sexually transmitted infections (STI) during the past 12 months, and HIV testing during lifetime. The interviews were conducted in French or Nengue Tongo (dominant language). The questionnaire was administered in face to face by trained interviewers (duration 45 minutes). It was adapted from a previous study describing the KABP of the general population of coastal French Guiana (in 2004, a random sample of 406 men was surveyed, using landline telephone lists) [5]. When the questions were similar in both questionnaires, the data were compared between boatmen and this previous KABP study [5]. The proportions were compared using a two sample proportion calculator in STATA (College Station, Texas).

\section{Results}

Overall, 127 boatmen were interrogated. All were male and the median age was 31 years (Inter Quartile Range: 25-38). Seventy three percent declared their principal residence was in Saint Laurent du Maroni; $67.7 \%$ were born in Suriname and 31.5\% in French Guiana;

${ }^{*}$ Corresponding author: Gaubert-Marechal Emilie, Corevih of Guyane, Hospital complex of Cayenne, Andrée Rosemon, Street Flamboyants, B.P 6006, 97306 Cayenne Cedex, France, Tel: 00594-594-394-812/00696-696-865-440; Fax: 00594.594.39.50.16; E-mail : emilie.gaubert-marechal@ch-cayenne.fr

Received September 18, 2012; Accepted November 16, 2012; Published November 21, 2012

Citation: Gaubert-Maréchal E, Jolivet A, Van-Melle A, Parriault Mc, Basurko C, et al. (2012) Knowledge, Attitudes, Beliefs and Practices on HIVIAIDS among Boatmen on the Maroni River: A Neglected Bridging Group? J AIDS Clin Res 3:181. doi:10.4172/2155-6113.1000181

Copyright: (C) 2012 Gaubert-Maréchal E, et al. This is an open-access article distributed under the terms of the Creative Commons Attribution License, which permits unrestricted use, distribution, and reproduction in any medium, provided the original author and source are credited. 
Citation: Gaubert-Maréchal E, Jolivet A, Van-Melle A, Parriault Mc, Basurko C, et al. (2012) Knowledge, Attitudes, Beliefs and Practices on HIV/AIDS among Boatmen on the Maroni River: A Neglected Bridging Group? J AIDS Clin Res 3:181. doi:10.4172/2155-6113.1000181

Page 2 of 3

$38.1 \%$ were French; $16.5 \%$ had never been to school; $29.9 \%$ were single, 3.2\% married, $55.9 \%$ married traditionally. Overall, $11.8 \%$ of boatmen declared having 2 "traditional" wives. Most boatmen had the knowledge of HIV transmission risk through unprotected sex (92.9\%). However, this proportion was lower than in the 406 men from the general population of coastal French Guiana surveyed in 2004 (97.6\%, $\mathrm{p}=0.01$ ). Erroneous beliefs persisted regarding transmission modes. For example, $23.6 \%$ of boatmen declared HIV could be transmitted by mosquitoes (vs. $15.5 \%$ in the general population of men, $\mathrm{p}=0.04$ ). Knowledge regarding prevention was lower: $72.4 \%$ of boatmen declared that condoms were an effective protection against HIV (vs. $95.2 \%$ in the general population of men, $\mathrm{p}<0.001)$. However, when asked whether female condoms were an effective protection against HIV, there was no difference between boatmen and the general population of men $(72 \%$ vs. $72 \%$, respectively $\mathrm{p}=0,98$ (Table 1 ).
The median age at first sex was 14 and $7.9 \%$ indicated having used a condom for their first sexual intercourse (vs. $27 \%$ in the general population of men, $\mathrm{p}<0.001)$. The proportion of men potentially exposed to HIV was higher among boatmen than in the general population. Indeed, $81 \%$ of boatmen declared having more than one sex partner in the 12 months before the study (vs. $21.1 \%$ of in the general population of men, $\mathrm{p}<0.001$ ); $50.4 \%$ declared having sex with a sex worker at least once in the past year (vs. $2.7 \%$ in the general population of men, $\mathrm{p}<0.001$ ) and $30.1 \%$ of boatmen declared at least one sexually transmitted infection in the past year (vs. $3.2 \%$ in the general population of men, $\mathrm{p}<0.001$ ). A lower proportion of boatmen declared never having had an HIV test in their life ( $23.6 \%$ vs. $38.6 \%$ in the general population of men, $\mathrm{p}=0,002$ ) (Table 1 ).

\begin{tabular}{|c|c|c|c|}
\hline & $\begin{array}{c}\text { Maroni Boatmen } \\
(n=127)\end{array}$ & $\begin{array}{l}\text { KABP-ORS Guiana } \\
\text { Men }(n=406)\end{array}$ & $\mathbf{p}$ \\
\hline & $\%$ & $\%$ & \\
\hline \multicolumn{4}{|l|}{$\begin{array}{l}\text { Knowledge of transmission routes } \\
\text { (\% of "yes") }\end{array}$} \\
\hline \multicolumn{4}{|l|}{ Circumstances of known transmission } \\
\hline During unprotected intercourse & 92.9 & 97.6 & 0.01 \\
\hline \multicolumn{4}{|l|}{ Circumstances without transmission } \\
\hline Through a mosquito bite & 23.6 & 15.5 & 0.04 \\
\hline By shaking the hand of an infected person & 4.8 & 2.6 & 0.21 \\
\hline During protected intercourse & 11.0 & 17.8 & 0.07 \\
\hline By drinking from the same glass or eating from the same plate as an infected person * & 7.9 & 8.6 & 0.80 \\
\hline AIDS is not a disease, it is a curse ${ }^{*}$ & 30.7 & - & \\
\hline \multicolumn{4}{|l|}{$\begin{array}{l}\text { Knowledge of protection strategies } \\
\text { (\% of « certainly or almost efficient » }\end{array}$} \\
\hline \multicolumn{4}{|l|}{ Efficient } \\
\hline Use of a male condom & 72.4 & 95.2 & $<0.001$ \\
\hline Use of a female condom & 71.7 & 71.8 & 0.98 \\
\hline \multicolumn{4}{|l|}{ Inefficient } \\
\hline Wash after intercourse & 18.1 & 14.7 & 0.36 \\
\hline Withdraw before the end of intercourse & 15.0 & 13.1 & 0.59 \\
\hline \multicolumn{4}{|l|}{ « complementary or alternatives » } \\
\hline Ask question to one's partner about her past sex life & 38.9 & 39.4 & 0.92 \\
\hline Ask partner to do an HIVIAIDS screening test & 56.7 & 67.0 & 0.03 \\
\hline Regularly do an HIVIAIDS screening test & 62.2 & 62.0 & 0.97 \\
\hline Choose partners that do not look sick ${ }^{* * *}$ & 26.0 & 35.4 & 0.05 \\
\hline Have intercourse with a limited range of different partners & 26.0 & 31.0 & 0.28 \\
\hline \multicolumn{4}{|l|}{ First intercourse } \\
\hline Median age at first intercourse (in years) & 14 & 15.4 & \\
\hline Declared use of condoms at first intercourse & $7.9 \%$ & $27.0 \%$ & $<0.001$ \\
\hline \multicolumn{4}{|c|}{ Sexual activity, recourse to paid sex and Sexually transmitted infections (STI) during the past 12 months } \\
\hline$\%$ having multiple partners ( $>1$ partners in the last 12 months) & $81.0 \%$ & $21.1 \%$ & $<0.001$ \\
\hline Use of condom during intercourse for those having multiple partners during the past 12 months & $92.2 \%$ & $81.1 \%$ & $<0.001$ \\
\hline$\%$ declaring use of condom during last intercourse & $57.7 \%$ & $25.4 \%$ & $<0.001$ \\
\hline At least one STI during the past 12 months & $30.1 \%$ & $3.2 \%$ & $<0.001$ \\
\hline$\%$ declaring having had sexual intercourse with sex workers (at least once) in the past 12 months ${ }^{*+* *}$ & $50.4 \%$ & $2.7 \%$ & $<0.001$ \\
\hline \multicolumn{4}{|l|}{ HIV testing during lifetime } \\
\hline$\%$ having never done an HIV test in their life & $23.6 \%$ & $38.6 \%$ & 0.002 \\
\hline
\end{tabular}

'The question was : "By drinking in the glass of an infected person in the KABP Guiana study

* The question was not used in the KABP coastal French Guiana Study

*** The question was : "by choosing partners correctly" in the KABP Guiana study

**+ The question was : " having paid for or been paid for sexual services » in the KABP Guiana study

Table 1: Comparison of main results from the boatmen study and the KABP-ORS Guiana 2004 study (Data from ORS Ile-de-France/ANRS-Knowledge, attitudes, beliefs and behaviors towards HIVIAIDS in the French West Indies and Guiana in 2004). 


\section{Discussion}

Although the present study has weaknesses, it is the first study describing a mobile population that cannot be sampled through probabilistic schemes.

Our study showed high rate of erroneous beliefs, sex with multiple partners and sex with sex workers among boatmen. Among erroneous beliefs it is interesting to notice that a $30.7 \%$ of the boatmen said that AIDS is not a disease, it is a curse. This question was added after discussions with local actors and by Treborg [2]. It was counter intuitive to observe that boatmen declared both having more STIs and using condom more frequently. This can be explained by the fact that if they had multiple partners and inconsistent condom use they could also get more STIs; another plausible explanation for this paradox would be that those with STIs may have subsequently used condoms more often.

The main limitations of the study were that boatmen interrogated may not have been representative of all boatmen on the Maroni and that the comparisons with the 2004 data compared results from different sampling designs and interview settings. Comparisons aside, the present study shows that a high proportion of Maroni boatmen is potentially at risk of acquiring and transmitting HIV. It therefore seems important to develop specific prevention efforts on this population group that has, up to now not been specifically targeted.

An additional possible explanation for the observed differences is that there are differences between the maroon populations in general (boatmen here were all maroons) and the population sampled by the KABP study (most of who were not maroons, and were not boatmen). Future studies in the general maroon population should disentangle the respective weight of boatman lifestyle, maroon culture, and socioeconomic factors in their relation to behaviour.
Cultural, lifestyle and personality factors are certainly important but access to prevention and care are definitely lower than in the rest of French Guiana. Although in depth study of attitudes towards risk should be initiated, a combination of specific preventive approaches should be prioritized in French Guiana. Thirty years after the first HIV case in French Guiana, the lack of knowledge on the epidemic drivers, maintained by fuzzy reasoning on the so-called 'generalized' epidemic, have slowed the adaptation of the response to the field. Boatmen may well represent a neglected bridging group between vulnerable sex workers and the general population on the Maroni. The present study opens a new avenue for prevention.

\section{Acknowledgments}

The study was funded by Réseau Kikiwi (Réseau Ville Hôpital pour le VIHSIDA, Maison des réseaux, Cayenne, Guyane, France).

\section{References}

1. Nacher M, Vantilcke V, Parriault MC, Van Melle A, Hanf M, et al. (2010) What is driving the HIV epidemic in French Guiana? Int J STD AIDS 21: 359-361.

2. Terborg J, Boven K (2000) Sexual behaviour and sexually transmitted diseases among maroon and indigenous populations in the hinterland of Suriname. ProHealth \& Primary Health Care Suriname, Paramaribo.

3. UNAIDS (2001) Population Mobility and AIDS: UNAIDS Technical update: February 2001. UNAIDS Information Centre, Geneva.

4. Lippman SA, Pulerwitz J, Chinaglia M, Hubbard A, Reingold A, et al. (2007) Mobility and its liminal context: Exploring sexual partnering among truck drivers crossing the Southern Brazilian border. Soc Sci Med 65: 2464-2473.

5. Halfen S, Fénies K, Ung B, Grémy I (2006) Les connaissances, attitudes croyances et comportements face au $\mathrm{VIH} / \mathrm{sida}$ aux Antilles et en Guyane en 2004. Rapport de l'ORS Île-de-France, Paris. 